\title{
Epstein Barr Virus: Potential Immune Selection in Associated Cancers
}

\author{
Nancy Raab-Traub* ${ }^{* \ddagger}$ and Rachel Edwards
}

Address: Lineberger Cancer Center, The University of North Carolina, Chapel Hill, NC 27599-7295

Email: Nancy Raab-Traub* - nrt@med.unc.edu

* Corresponding author $¥$ Presenting author

from 2005 International Meeting of The Institute of Human Virology

Baltimore, USA, 29 August - 2 September 2005

Published: 8 December 2005

Retrovirology 2005, 2(Suppl I):S49 doi:I0.1 I86/I742-4690-2-SI-S49

The EBV latent membrane protein 1 (LMP1) is expressed in most of the EBV-associated malignancies including nasopharyngeal carcinoma (NPC), Hodgkin's Lymphoma, and immunosuppression-associated lymphoma. have been identified by distinguishing amino acids. We have identified seven sequence variants of LMP1 that can be distinguished using a heteroduplex tracking assay and have determined that most healthy individuals are infected with multiple strains of EBV. Striking differences were found between NPC and matching blood samples with one specific variant, China 1, prevalent in NPC samples and multiple other variants of LMP1 prevalent in the blood samples. The possible selection against some strains appearing in the tumor was highly significant with a $p<0.0001$. Many of the LMP1 variants had changes in predicted HLA epitopes of various restrictions. The potential negative selection of the immune system on strains detected in the blood would be reflected in the striking predominance of the China 1 strain in the tumors. In lymphoma samples, changes were also frequently detected in known HLA-restricted epitopes of EBNA3A, 3B, or 3C, in addition to LMP1. Variation in potential immune recognition could contribute to the development of EBV-associated diseases in distinct populations and individuals. 